\title{
New Oleanene Glycosides from the Leaves of Acanthopanax japonicus
}

\author{
Sang-Yong PARK, ${ }^{*, a}$ Chang-Soo Yook, ${ }^{a}$ and Toshihiro NOHARA ${ }^{b}$ \\ ${ }^{a}$ Department of Pharmacognosy, College of Pharmacy, KyungHee University; 1 Hoegidong, Dongdaemungu, Seoul, \\ 130-702, Korea: and ${ }^{b}$ Faculty of Pharmaceutical Sciences, Kumamoto University; 5-1 Oe-honmachi, Kumamoto \\ 862-0973, Japan. Received April 15, 2005; accepted May 24, 2005
}

\begin{abstract}
The structures of 6 new oleanene glycosides (1-6) isolated from the leaves of Acanthopanax japonicus Franch $e$ t. SaVART (Araliaceae) were elucidated by mass, 1D, and 2D NMR spectroscopy. The structures of $1-6$ were established as 28 - $O$-[ $\alpha$-L-rhamnopyranosyl-( $1 \rightarrow 4)$ - $\beta$-D-glucopyranosyl-( $1 \rightarrow 6)-\beta$-D-glucopyranosyl] ester of $3 \beta, 23$-dihydroxy-olean-12-en-28,29-dioic acid, $3 \beta, 30$-dihydroxy-olean-12-en-23,28-dioic acid, $3 \beta, 29$-dihydroxyolean-12-en-23,28-dioic acid (=dianic aicd), 3 $\beta$-dihydroxy-olean-12-en-23,28-dioic acid (=gypsogenic acid), $3 \beta, 29$-dihydroxy-23-oxo-olean-12-en-28-oic acid, and $3 \beta$-hydroxy-23-oxo-olean-12-en-28,29-dioic acid, designated acanjaposide D (1), E (2), F (3), G (4), H (5), and I (6), respectively.
\end{abstract}

Key words Acanthopanax japonicus; Araliaceae; oleanene-type glycoside; acanjaposide D-I

The Acanthopanax genus is an important herbal drug used to strengthen sinew and bone, and to disperse water swelling in traditional oriental medicine. The leaves and roots of this genus, as well as stem bark, have been generally taken as a health food in Korea. Earlier, ${ }^{1)}$ we reported on the isolation and characterization of nor-oleanene type glycosides (acanjaposides A-C) from the methanolic extract of the leaves of Acanthopanx japonicus Franch et. SAVART (Araliaceae) distributed in Japan as an endemic species in our course of phytochemical studies on the genus of Acanthopanax. Further studies on this extract led to the isolation of 6 new glycosides of oleanene derivatives, based on their spectroscopic data including HR-MS, ${ }^{1} \mathrm{H}$ - and ${ }^{13} \mathrm{C}-\mathrm{NMR}$ spectroscopic data, designated acanjaposide D (1), E (2), F (3), G (4), H (5), and I (6).

Compound 1, acanjaposide $\mathrm{D}$, was obtained as a white amorphous powder of molecular formula $\mathrm{C}_{48} \mathrm{H}_{76} \mathrm{O}_{20}$ as determined by positive HR-FAB-MS. It exhibited a molecular ion peak at $\mathrm{m} / \mathrm{z} 995.4826$ due to $[\mathrm{M}+\mathrm{Na}]^{+}$(Calcd for
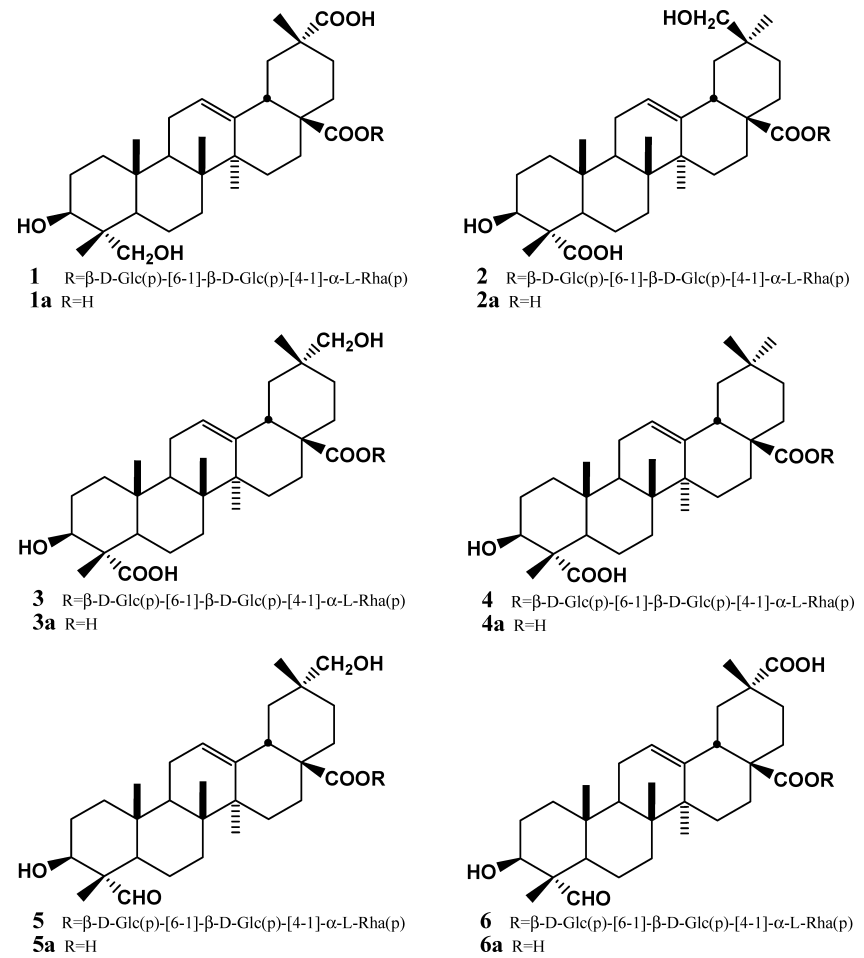

$\mathrm{C}_{48} \mathrm{H}_{76} \mathrm{O}_{20} \cdot \mathrm{Na}$ : 995.4828). The ${ }^{1} \mathrm{H}-\mathrm{NMR}$ spectrum of 1 revealed signals for 5 tertiary methyl groups $[\delta 1.00,1.05$, $1.13,1.18,1.43(3 \mathrm{H}, \mathrm{s})]$, a secondary methyl group $[\delta 1.68$ $(3 \mathrm{H}, \mathrm{d}, J=6.1 \mathrm{~Hz})], 1$ trisubstituted olefinic proton $[\delta 5.49$ $(1 \mathrm{H}$, br s $)]$, a methine proton $[\delta 3.30(1 \mathrm{H}, \mathrm{dd}, J=3.2$, $13.7 \mathrm{~Hz})]$ and 3 anomeric protons $[\delta 6.27(1 \mathrm{H}, \mathrm{d}, J=8.6 \mathrm{~Hz})$, $5.82(1 \mathrm{H}, \mathrm{s}), 4.98(1 \mathrm{H}, \mathrm{d}, J=7.9 \mathrm{~Hz})]$. The ${ }^{13} \mathrm{C}-\mathrm{NMR}$ spectrum of compound 1 showed 48 carbon signals, of which 30 were assigned to a triterpene moiety and 18 to three sugar moieties. Alkaline hydrolysis of $\mathbf{1}$ yielded $\mathbf{1 a}$ as a white amorphous powder, which had a molecular ion peak at $\mathrm{m} / \mathrm{z}$ 502 and characteristic fragment ion peaks at $\mathrm{m} / \mathrm{z} 278$ and 233 (produced by a retro Diels-Alder cleavage of C-ring in its EIMS). ${ }^{2,3)}$ The ${ }^{1} \mathrm{H}-\mathrm{NMR}$ spectrum of $\mathbf{1 a}$ showed signals for 5 tertiary methyl groups $[\delta 0.97,1.05,1.05,1.24,1.57(3 \mathrm{H}$, $\mathrm{s})$ ], an olefinic proton ( $\delta 5.58 \mathrm{brs}$ ), an oxygen-bearing methine proton $[\delta 4.18(1 \mathrm{H}, \mathrm{dd}, J=5.5,11.0 \mathrm{~Hz})], 2$ oxygenbearing methylene protons $[\delta 3.71(1 \mathrm{H}, \mathrm{d}, J=11.0 \mathrm{~Hz})$ and $4.16(1 \mathrm{H}, \mathrm{d}, J=11.0 \mathrm{~Hz})]$ and a methine proton $[\delta 3.44(1 \mathrm{H}$, dd, $J=3.7,13.7 \mathrm{~Hz}$ )]. The ${ }^{13} \mathrm{C}-\mathrm{NMR}$ spectrum of $\mathbf{1 a}$ showed 30 carbon signals, assignable to 5 methyl carbons, 11 methylene carbons, 5 methine carbons, and 9 quaternary carbons by DEPT and HMQC spectra. Among 2 carbonyl groups, C$28(\delta 180.6)$ showed a glycosylation-induced shift (Table 1$)$. These findings suggested that aglycone $1 \mathrm{a}$ was a $\Delta^{12}$-unsaturated oleanene glycoside of $\mathrm{C}_{30} \mathrm{H}_{46} \mathrm{O}_{6}$, and that $\mathbf{1}$ was a $28-\mathrm{O}$ monodesmoside with tri-saccharides. ${ }^{4)}$ Acid hydrolysis of $\mathbf{1}$ afforded the sugar moieties of L-rhamnose and D-glucose which were identified by GLC. ${ }^{1)}$ The identity of the single sugar moiety and the sequence of the oligosaccharide chain were determined by HMBC, HMQC and ${ }^{1} \mathrm{H}-{ }^{1} \mathrm{H}$ COSY. The 2 glucose moieties were ascertained to have $\beta$-anomeric configuration from ${ }^{3} J_{\mathrm{H} 1-\mathrm{H} 2}$ coupling constants $[\delta 6.27(1 \mathrm{H}$, d, $\left.J=8.6 \mathrm{~Hz}, \mathrm{Glc} \mathrm{H}-1), 4.98\left(1 \mathrm{H}, \mathrm{d}, J=7.9 \mathrm{~Hz}, \mathrm{Glc}^{\prime} \mathrm{H}-1\right)\right]$, and the anomeric configuration of the rhamnose moiety was determined to be the $\alpha$-form from ${ }^{13} \mathrm{C}$-NMR chemical shifts of the C-3 and C-5 rhamnosyl moieties ( $\delta 72.7,70.3$, respectively). ${ }^{5)}$ Therefore, the sugar moiety was determined to be 28 - $O$ - $\alpha$-L-rhamnopyranosyl-( $1 \rightarrow 4)$ - $\beta$-D-glucopyranosyl$(1 \rightarrow 6)]-\beta$-D-glucopyranosyl ester, which is common in the Acanthopanax species. ${ }^{6-12)}$ The proton signal at $\delta 4.18(1 \mathrm{H}$, dd, $J=5.2,11.0 \mathrm{~Hz}$ ) was assigned to $\mathrm{H}-3$ by $2 \mathrm{D}-\mathrm{NMR}$, and correlated with $\delta_{\mathrm{C}} 73.6$ in the HMQC spectrum and the cross 
peak between H-24 $(\delta$ 1.05) and C-3 $(\delta$ 73.6) in the HMBC spectrum. The hydroxyl group at $\mathrm{C}-3$ appeared to have a $\beta$ configuration based on its coupling constants in the ${ }^{1} \mathrm{H}-\mathrm{NMR}$ spectrum. ${ }^{13)}$ The proton signals at $\delta 3.71$ and 4.16 were found to originate from the $\mathrm{H}-23$ of oxymethylene group at C-4 based on the HMBC correlation between H-24 ( $\delta$ 1.05) and $\mathrm{C}-23(\delta 68.1)$, and the functional group was concluded to be in the $\alpha$-configuration according to ${ }^{13} \mathrm{C}$-NMR spectral data $\left(\delta_{\mathrm{C}-23} 68.1, \delta_{\mathrm{C}-24} 13.1\right) .{ }^{1)}$ Two carboxylic carbon signals at 180.6 and 181.0 were assigned to $\mathrm{C}-28$ and $\mathrm{C}-29$, respectively, by their HMBC and HMQC. These assignments were confirmed by NOESY spectrum, which showed a NOE correlation between the tertiary methyl proton signal $(\delta 1.43, \mathrm{H}$ 30 ) and the methine proton signal at 3.30 (dd, $J=3.7$, 13.7 Hz, H-18). This assignment was ascertained by comparison with the ${ }^{13} \mathrm{C}-\mathrm{NMR}$ spectrum of serratagenic acid. ${ }^{14,15)}$ Accordingly, the structure of $\mathbf{1 a}$ was determined as 3 $\beta, 23$-dihydroxy-olean-12-ene-28,29-dioic acid.

Therefore, 1 was determined to be 3 $\beta, 23$-dihydroxy-olean12-ene-28,29-dioic acid 28- $O$ - $\alpha$-L-rhamnopyranosyl- $(1 \rightarrow 4)$ $\beta$-D-glucopyranosyl-( $1 \rightarrow 6)]$ - $\beta$-D-glucopyranoside, and was termed acanjaposide $\mathrm{D}$.

The second new compound (2), called acanjaposide E, showed a quasimolecular ion peak at $\mathrm{m} / \mathrm{z} 996$ due to $[\mathrm{M}+\mathrm{Na}]^{+}$by FAB-MS and its ${ }^{13} \mathrm{C}-\mathrm{NMR}$ spectrum showed the presence of 48 carbon signals $\left(\mathrm{CH}_{3} \times 6,-\mathrm{CH}_{2}-\times 11\right.$, $>\mathrm{CH}-\times 3,>\mathrm{C}<\times 6, \mathrm{CH}_{2}-\mathrm{O} \times 3,>\mathrm{CH}-\mathrm{O} \times 13, \mathrm{O}-\mathrm{CH}-\mathrm{O} \times$ 3 , $>\mathrm{C}=\mathrm{CH}-\times 1$, and $\mathrm{CO} \times 2$ ). These data indicated that acanjaposide $\mathrm{E}$ (2) had the molecular formula $\mathrm{C}_{48} \mathrm{H}_{76} \mathrm{O}_{20}$. This assignment is consistent with HR-FAB-MS spectrum showing $m / z 995.4821$ (Calcd for $\mathrm{C}_{48} \mathrm{H}_{76} \mathrm{O}_{20} \cdot \mathrm{Na}$ : 995.4828). Alkaline hydrolysis of $\mathbf{2}$ afforded $\mathbf{2 a}$, a novel aglycone, and we verified by sugar analysis and by ${ }^{1} \mathrm{H}$ - and ${ }^{13} \mathrm{C}-\mathrm{NMR}$ that the sugar moiety was the same as that of 1 . The HR-FAB-MS spectrum of $\mathbf{2 a}$ had a quasimolecular ion peak at $\mathrm{m} / \mathrm{z}$ 525.3187 due to $[\mathrm{M}+\mathrm{Na}]^{+}$, which corresponds to the molecular formula $\mathrm{C}_{30} \mathrm{H}_{46} \mathrm{O}_{6}$. Moreover, the ${ }^{13} \mathrm{C}-\mathrm{NMR}$ resonance signals attributable to the carbons of the A, B, C and D-rings of the aglycone (2a) matched those of gypsogenic acid (4a) (Table 1). Furthermore, although the ${ }^{13} \mathrm{C}-\mathrm{NMR}$ spectrum of the E-ring of $\mathbf{2 a}$ differed from that of nipponoside $\mathrm{D}$ (3 $\beta, 23,29$-trihydroxy-olean-12-en-28-oic acid glycoside) previously isolated from this species, ${ }^{1)}$ the observed ${ }^{13} \mathrm{C}$-NMR data of C-29 and C-30 were in good agreement with methyl querotaroate $^{15)}$ and spinoside $\mathrm{C} 2{ }^{9}{ }^{9}$ The above results and their ${ }^{13} \mathrm{C}$-NMR data indicated that $\mathbf{2 a}$ was 30 -hydroxygypsogenic acid with an axial oxymethylene group at C-20. This conclusion was confirmed by 2D-NMR and NOESY, which showed a NOE correlation between $\mathrm{H}-30 a[\delta 3.92(1 \mathrm{H}$, $J=10.4 \mathrm{~Hz})]$ to $\mathrm{H}-18[\delta 3.44(1 \mathrm{H}, \mathrm{dd}, J=6.4,11.9 \mathrm{~Hz})]$ and $\mathrm{H}-24[\delta 1.64(3 \mathrm{H}, \mathrm{s})]$ to $\mathrm{H}-25[\delta 0.96(3 \mathrm{H}, \mathrm{s})]$. Thus, 2 was identified as 3 $\beta, 30$-dihydroxy-olean-12-en-23,28-dioic acid (30-hydroxy gypsogenic acid) 28- $O-\alpha$-L-rhamnopyranosyl$(1 \rightarrow 4)$ - $\beta$-D-glucopyranosyl-( $1 \rightarrow 6)]-\beta$-D-glucopyranoside, and called acanjaposide $\mathrm{E}$.

Compound 3, acanjaposide $\mathrm{F}$, had a quasimolecular ion peak at 995.4835 due to $[\mathrm{M}+\mathrm{Na}]^{+}$in the HR-FAB-MS, corresponding to the molecular formula $\mathrm{C}_{48} \mathrm{H}_{76} \mathrm{O}_{20}$ (Calcd for $\mathrm{C}_{48} \mathrm{H}_{76} \mathrm{O}_{20} \cdot \mathrm{Na}:$ 995.4828). The ${ }^{13} \mathrm{C}-\mathrm{NMR}$ spectrum of 3 showed 48 carbon signals, 30 of which were assigned to the aglycone moiety and 18 to an oligosaccharide, which ap- peared to be the same as that of 1 based on $1 \mathrm{D}$ and 2D-NMR data which included ${ }^{1} \mathrm{H}-{ }^{1} \mathrm{H}$ COSY and HMBC experiments. Sugar analysis confirmed that the oligosaccharide was 28-O$\alpha$-L-rhamnopyranosyl-( $1 \rightarrow 4)$ - $\beta$-D-glucopyranosyl-( $1 \rightarrow 6)]-\beta$ D-glucopyranoside, like that of acanjaposide E (1).

When compound 3 was subjected to alkali hydrolysis with $3 \% \mathrm{KOH}$ it yielded the sapogenin (3a) which contained five tertiary methyl groups $[\delta 0.98,1.04,1.22,1.27,1.64(3 \mathrm{H}$, $\mathrm{s})]$, one oxymethylene group $[\delta 3.59(2 \mathrm{H}, \mathrm{s})]$, one oxymethine proton $[\delta 4.68(1 \mathrm{H}, \mathrm{dd}, J=7.9,7.9 \mathrm{~Hz})]$, a tri-substituted olefinic proton $[\delta 5.54(1 \mathrm{H}$, br s $)]$, and a methine proton $[\delta 3.42(1 \mathrm{H}, \mathrm{dd}, J=3.4,13.6 \mathrm{~Hz})]$. The molecular formula of 3a, $\mathrm{C}_{30} \mathrm{H}_{46} \mathrm{O}_{6}$, was established by positive FAB-MS $\left(m / z 525,[\mathrm{M}+\mathrm{Na}]^{+}\right)$and EI-MS, which showed a molecular ion at $\mathrm{m} / \mathrm{z} 486$ and fragments at $\mathrm{m} / \mathrm{z} 264$ and 219 produced by retro Diels-Alder cleavage of the C-ring in olean-12-ene.

NMR analysis and MS spectral data demonstrated that compound 3a was a 33,29-dihydroxyl-olean12-en-23,28dioic acid, which is an analogue of gypsogenic acid with a hydroxyl group attached at C-29 (=29-hydroxyl gypsogenic acid), called dianic acid. ${ }^{16)}$ Therefore, compound $\mathbf{3}$ was identified as 3 $\beta, 29$-hydroxy-olean-12-en-23,28-dioic acid 28-O$\alpha$-L-rhamnopyranosyl-( $1 \rightarrow 4)$ - $\beta$-D-glucopyranosyl- $(1 \rightarrow 6)]-\beta$ D-glucopyranoside, and termed acanjaposide $\mathrm{F}$.

In the HR-FAB-MS, compound 4 exhibited a quasimolecular ion at $\mathrm{m} / \mathrm{z} 979.4905$ due to $[\mathrm{M}+\mathrm{Na}]^{+}$corresponding to the molecular formula $\mathrm{C}_{48} \mathrm{H}_{76} \mathrm{O}_{19}$. Since this compound was suspected to be a triterpenoidal saponin due to the positive Lieberman-Burchard reaction and by the ${ }^{13} \mathrm{C}-\mathrm{NMR}$ spectral analysis, 4 was also subjected to alkali hydrolysis and yielded the aglycone $\mathbf{4 a}$, which showed a quasimolecular ion peak at 509 due to $[\mathrm{M}+\mathrm{Na}]^{+}$by positive $\mathrm{FAB}-\mathrm{MS}$, and a molecular ion $\left(\mathrm{C}_{30} \mathrm{H}_{46} \mathrm{O}_{6}\right)$ at $\mathrm{m} / z 486$ along with fragments at $m / z 248$ and 203 in EI-MS. Consequently, 4a was identified as gypsogenic acid based on ${ }^{1} \mathrm{H}$ - and ${ }^{13} \mathrm{C}-\mathrm{NMR}$ spectral data (Table 1). ${ }^{16)}$ It was ascertained by sugar analysis and its NMR spectral data that the sugar moiety of $\mathbf{4}$ was the same as that of $\mathbf{1}$. Thus, compound $\mathbf{4}$ was identified as gypsogenic acid $28-O$ - $\alpha$-L-rhamnopyranosyl- $(1 \rightarrow 4)-\beta$-D-glucopyranosyl$(1 \rightarrow 6)]$ - $\beta$-D-glucopyranoside, and called acanjaposide G.

Acanjaposide $\mathrm{H}(\mathbf{5})$, a white powder, produced quasimolecular ion at $m / z 979.4884$ due to $[\mathrm{M}+\mathrm{Na}]^{+}$in the positive HR-FAB-MS, and its molecular formula was determined to be $\mathrm{C}_{48} \mathrm{H}_{76} \mathrm{O}_{19}$ from this MS and its NMR data (Calcd for $\mathrm{C}_{48} \mathrm{H}_{76} \mathrm{O}_{19} \mathrm{Na}$ : 979.4878).

${ }^{1} \mathrm{H}$ - and ${ }^{13} \mathrm{C}-\mathrm{NMR}$ spectrum of $\mathbf{5}$ exhibited three anomeric proton signals at $\delta 6.25(\mathrm{~d}, J=7.9 \mathrm{~Hz}), 4.99(\mathrm{~d}, J=7.9 \mathrm{~Hz})$, and 5.85 (brs) and anomeric carbon signals at $\delta$ 95.6, 104.9, and 102.7 , respectively. The ${ }^{13} \mathrm{C}-\mathrm{NMR}$ spectrum showed 48 carbon signals of which 30 were assigned to the aglycone moiety and 18 to the oligosaccharide. The 1D and 2D-NMR data including ${ }^{1} \mathrm{H}-{ }^{1} \mathrm{H}$ COSY and HMBC experiments, and sugar analysis allowed the assignments of its sugar components and sequence to $28-O-\alpha$-L-rhamnopyranosyl- $(1 \rightarrow 4)-\beta$ D-glucopyranosyl-( $1 \rightarrow 6)]$ - $\beta$-D-glucopyranoside, as for acanjaposide E (1).

Alkali hydrolysis of $\mathbf{5}$ afforded the novel sapogenin (5a), which showed 5 tertiary methyl groups $[\delta 0.90,1.00,1.22$, $1.30,1.35($ all $3 \mathrm{H}, \mathrm{s})], 1$ oxymethylene proton $[\delta 3.60(2 \mathrm{H}$, s)], 1 oxymethine proton [ $\delta 4.06(1 \mathrm{H}, \mathrm{dd}, J=7.3,8.6 \mathrm{~Hz})]$, a tri-substituted olefinic proton $[\delta 5.23(1 \mathrm{H}$, br s) $]$, and a me- 
Table 1. ${ }^{13} \mathrm{C}-\mathrm{NMR}$ Data for Compounds $\mathbf{1}-\mathbf{6}$ and Their Algycone in Pyridine- $d_{5}(125 \mathrm{MHz})$

\begin{tabular}{|c|c|c|c|c|c|c|c|c|c|c|c|c|}
\hline $\mathrm{C}$ & 1 & $1 \mathrm{a}$ & 2 & $2 a$ & 3 & $3 \mathbf{a}$ & 4 & $4 a$ & 5 & $5 \mathbf{a}$ & 6 & $6 a$ \\
\hline 1 & $38.8 \mathrm{t}$ & $38.8 \mathrm{t}$ & $39.1 \mathrm{t}$ & $39.1 \mathrm{t}$ & $39.1 \mathrm{t}$ & $39.0 \mathrm{t}$ & $39.0 \mathrm{t}$ & $39.0 \mathrm{t}$ & $38.5 \mathrm{t}$ & $38.5 \mathrm{t}$ & $38.5 \mathrm{t}$ & $38.5 \mathrm{t}$ \\
\hline 2 & $27.5 \mathrm{t}$ & $27.6 \mathrm{t}$ & $27.8 \mathrm{t}$ & $27.8 \mathrm{t}$ & $27.8 \mathrm{t}$ & $27.8 \mathrm{t}$ & $27.7 \mathrm{t}$ & $27.7 \mathrm{t}$ & $27.1 \mathrm{t}$ & $27.0 \mathrm{t}$ & $27.0 \mathrm{t}$ & $27.0 \mathrm{t}$ \\
\hline 3 & $73.4 \mathrm{~d}$ & $73.6 \mathrm{~d}$ & $75.5 \mathrm{~d}$ & $75.5 \mathrm{~d}$ & $75.5 \mathrm{~d}$ & $75.5 \mathrm{~d}$ & $75.5 \mathrm{~d}$ & $75.5 \mathrm{~d}$ & $71.7 \mathrm{~d}$ & $71.7 \mathrm{~d}$ & $71.6 \mathrm{~d}$ & $71.6 \mathrm{~d}$ \\
\hline 4 & $42.8 \mathrm{~s}$ & $42.9 \mathrm{~s}$ & $54.5 \mathrm{~s}$ & $54.5 \mathrm{~s}$ & $54.4 \mathrm{~s}$ & $54.4 \mathrm{~s}$ & $54.4 \mathrm{~s}$ & $54.4 \mathrm{~s}$ & $56.3 \mathrm{~s}$ & $56.3 \mathrm{~s}$ & $56.3 \mathrm{~s}$ & $56.3 \mathrm{~s}$ \\
\hline 5 & $48.5 \mathrm{~d}$ & $48.7 \mathrm{~d}$ & $51.9 \mathrm{~d}$ & $52.0 \mathrm{~d}$ & $51.9 \mathrm{~d}$ & $52.0 \mathrm{~d}$ & $51.9 \mathrm{~d}$ & $51.9 \mathrm{~d}$ & $48.0 \mathrm{~d}$ & $48.0 \mathrm{~d}$ & $47.9 \mathrm{~d}$ & $48.0 \mathrm{~d}$ \\
\hline 6 & $18.5 \mathrm{t}$ & $18.6 \mathrm{t}$ & $21.7 \mathrm{t}$ & $21.7 \mathrm{t}$ & $21.7 \mathrm{t}$ & $21.7 \mathrm{t}$ & $21.7 \mathrm{t}$ & $21.7 \mathrm{t}$ & $21.1 \mathrm{t}$ & $21.0 \mathrm{t}$ & $21.0 \mathrm{t}$ & $21.0 \mathrm{t}$ \\
\hline 7 & $32.8 \mathrm{t}$ & $32.9 \mathrm{t}$ & $33.0 \mathrm{t}$ & $33.0 \mathrm{t}$ & $32.9 \mathrm{t}$ & $33.0 \mathrm{t}$ & $32.9 \mathrm{t}$ & $33.0 \mathrm{t}$ & $32.5 \mathrm{t}$ & $32.7 \mathrm{t}$ & $32.4 \mathrm{t}$ & $32.5 \mathrm{t}$ \\
\hline 8 & $39.9 \mathrm{~s}$ & $39.8 \mathrm{~s}$ & $40.3 \mathrm{~s}$ & $40.0 \mathrm{~s}$ & $40.3 \mathrm{~s}$ & $40.1 \mathrm{~s}$ & $40.2 \mathrm{~s}$ & $40.1 \mathrm{~s}$ & $40.2 \mathrm{~s}$ & $40.1 \mathrm{~s}$ & $40.2 \mathrm{~s}$ & $40.1 \mathrm{~s}$ \\
\hline 9 & $48.1 \mathrm{~d}$ & $48.1 \mathrm{~d}$ & $48.4 \mathrm{~d}$ & $48.4 \mathrm{~d}$ & $48.4 \mathrm{~d}$ & $48.4 \mathrm{~d}$ & $48.3 \mathrm{~d}$ & $48.4 \mathrm{~d}$ & $47.7 \mathrm{~d}$ & $47.7 \mathrm{~d}$ & $47.6 \mathrm{~d}$ & $47.7 \mathrm{~d}$ \\
\hline 10 & $37.2 \mathrm{~s}$ & $37.2 \mathrm{~s}$ & $36.9 \mathrm{~s}$ & $36.9 \mathrm{~s}$ & $36.9 \mathrm{~s}$ & $36.9 \mathrm{~s}$ & $36.8 \mathrm{~s}$ & $36.7 \mathrm{~s}$ & $36.1 \mathrm{~s}$ & $36.2 \mathrm{~s}$ & $36.1 \mathrm{~s}$ & $36.2 \mathrm{~s}$ \\
\hline 11 & $23.8 \mathrm{t}$ & $23.9 \mathrm{t}$ & $23.8 \mathrm{t}$ & $23.8 \mathrm{t}$ & $23.9 \mathrm{t}$ & $23.8 \mathrm{t}$ & $23.8 \mathrm{t}$ & $23.9 \mathrm{t}$ & $23.8 \mathrm{t}$ & $23.8 \mathrm{t}$ & $23.7 \mathrm{t}$ & $23.8 \mathrm{t}$ \\
\hline 12 & $123.5 \mathrm{~d}$ & $123.3 \mathrm{~d}$ & $122.9 \mathrm{~d}$ & $122.5 \mathrm{~d}$ & $122.8 \mathrm{~d}$ & $122.4 \mathrm{~d}$ & $122.7 \mathrm{~d}$ & $122.4 \mathrm{~d}$ & $122.6 \mathrm{~d}$ & $122.3 \mathrm{~d}$ & $123.2 \mathrm{~d}$ & $122.9 \mathrm{~d}$ \\
\hline 13 & $143.6 \mathrm{~s}$ & $144.3 \mathrm{~s}$ & $144.1 \mathrm{~s}$ & $144.8 \mathrm{~s}$ & $144.4 \mathrm{~s}$ & $145.0 \mathrm{~s}$ & $144.1 \mathrm{~s}$ & $144.9 \mathrm{~s}$ & $144.4 \mathrm{~s}$ & $145.1 \mathrm{~s}$ & $143.7 \mathrm{~s}$ & $144.1 \mathrm{~s}$ \\
\hline 14 & $42.1 \mathrm{~s}$ & $42.2 \mathrm{~s}$ & $42.2 \mathrm{~s}$ & $42.2 \mathrm{~s}$ & $42.1 \mathrm{~s}$ & $42.1 \mathrm{~s}$ & $42.1 \mathrm{~s}$ & $42.2 \mathrm{~s}$ & $42.2 \mathrm{~s}$ & $42.2 \mathrm{~s}$ & $42.1 \mathrm{~s}$ & $42.2 \mathrm{~s}$ \\
\hline 15 & $28.2 \mathrm{t}$ & $28.3 \mathrm{t}$ & $28.4 \mathrm{t}$ & $28.4 \mathrm{t}$ & $28.3 \mathrm{t}$ & $28.3 \mathrm{t}$ & $28.2 \mathrm{t}$ & $28.3 \mathrm{t}$ & $28.3 \mathrm{t}$ & $28.3 \mathrm{t}$ & $28.1 \mathrm{t}$ & $28.2 \mathrm{t}$ \\
\hline 16 & $23.4 \mathrm{t}$ & $23.9 \mathrm{t}$ & $23.7 \mathrm{t}$ & $24.0 \mathrm{t}$ & $23.4 \mathrm{t}$ & $23.7 \mathrm{t}$ & $23.3 \mathrm{t}$ & $23.7 \mathrm{t}$ & $23.4 \mathrm{t}$ & $23.7 \mathrm{t}$ & $23.4 \mathrm{t}$ & $23.8 \mathrm{t}$ \\
\hline 17 & $47.0 \mathrm{~s}$ & $46.6 \mathrm{~s}$ & $47.2 \mathrm{~s}$ & $46.7 \mathrm{~s}$ & $47.5 \mathrm{~s}$ & $47.1 \mathrm{~s}$ & $47.0 \mathrm{~s}$ & $46.6 \mathrm{~s}$ & $47.5 \mathrm{~s}$ & $47.1 \mathrm{~s}$ & $46.9 \mathrm{~s}$ & $46.6 \mathrm{~s}$ \\
\hline 18 & $40.8 \mathrm{~d}$ & $41.1 \mathrm{~d}$ & $41.3 \mathrm{~d}$ & $41.6 \mathrm{~d}$ & $41.1 \mathrm{~d}$ & $41.4 \mathrm{~d}$ & $41.6 \mathrm{~d}$ & $42.0 \mathrm{~d}$ & $41.2 \mathrm{~d}$ & $41.5 \mathrm{~d}$ & $40.8 \mathrm{~d}$ & $41.1 \mathrm{~d}$ \\
\hline 19 & $40.8 \mathrm{t}$ & $41.0 \mathrm{t}$ & $41.7 \mathrm{t}$ & $42.0 \mathrm{t}$ & $41.0 \mathrm{t}$ & $41.3 \mathrm{t}$ & $46.2 \mathrm{t}$ & $46.5 \mathrm{t}$ & $40.9 \mathrm{t}$ & $41.2 \mathrm{t}$ & $40.8 \mathrm{t}$ & $41.0 \mathrm{t}$ \\
\hline 20 & $42.3 \mathrm{~s}$ & $42.6 \mathrm{~s}$ & $35.7 \mathrm{~d}$ & $35.9 \mathrm{~d}$ & $36.4 \mathrm{~d}$ & $36.6 \mathrm{~d}$ & $30.7 \mathrm{~d}$ & $31.0 \mathrm{~d}$ & $36.4 \mathrm{~s}$ & $36.6 \mathrm{~s}$ & $42.3 \mathrm{~s}$ & $42.6 \mathrm{~s}$ \\
\hline 21 & $29.1 \mathrm{t}$ & $29.3 \mathrm{t}$ & $29.5 \mathrm{t}$ & $29.6 \mathrm{t}$ & $28.8 \mathrm{t}$ & $29.1 \mathrm{t}$ & $33.9 \mathrm{t}$ & $34.2 \mathrm{t}$ & $28.8 \mathrm{t}$ & $29.1 \mathrm{t}$ & $29.1 \mathrm{t}$ & $29.3 \mathrm{t}$ \\
\hline 22 & $31.7 \mathrm{t}$ & $32.4 \mathrm{t}$ & $32.3 \mathrm{t}$ & $33.1 \mathrm{t}$ & $32.0 \mathrm{t}$ & $32.7 \mathrm{t}$ & $32.4 \mathrm{t}$ & $33.2 \mathrm{t}$ & $32.0 \mathrm{t}$ & $32.6 \mathrm{t}$ & $31.6 \mathrm{t}$ & $32.3 \mathrm{t}$ \\
\hline 23 & $67.9 \mathrm{t}$ & $68.1 \mathrm{t}$ & $180.7 \mathrm{~s}$ & $180.7 \mathrm{~s}$ & $180.7 \mathrm{~s}$ & $180.6 \mathrm{~s}$ & $180.7 \mathrm{~s}$ & $180.3 \mathrm{~s}$ & $207.3 \mathrm{q}$ & $207.3 \mathrm{q}$ & $207.4 \mathrm{q}$ & $207.3 \mathrm{q}$ \\
\hline 24 & $13.1 \mathrm{q}$ & $13.1 \mathrm{q}$ & $12.3 \mathrm{q}$ & $12.3 \mathrm{q}$ & $12.3 \mathrm{q}$ & $12.2 \mathrm{q}$ & $12.2 \mathrm{q}$ & $12.3 \mathrm{q}$ & $9.7 \mathrm{q}$ & $9.7 \mathrm{q}$ & $9.6 \mathrm{q}$ & $9.6 \mathrm{q}$ \\
\hline 25 & $16.1 \mathrm{q}$ & $16.0 \mathrm{q}$ & $16.1 \mathrm{q}$ & $16.0 \mathrm{q}$ & $16.1 \mathrm{q}$ & $16.0 \mathrm{q}$ & $16.1 \mathrm{q}$ & $16.0 \mathrm{q}$ & $15.8 \mathrm{q}$ & $15.7 \mathrm{q}$ & $15.8 \mathrm{q}$ & $15.7 \mathrm{q}$ \\
\hline 26 & $17.6 \mathrm{q}$ & $17.5 \mathrm{q}$ & $17.5 \mathrm{q}$ & $17.4 \mathrm{q}$ & $17.5 \mathrm{q}$ & $17.3 \mathrm{q}$ & $17.4 \mathrm{q}$ & $17.3 \mathrm{q}$ & $17.5 \mathrm{q}$ & $17.4 \mathrm{q}$ & $17.4 \mathrm{q}$ & $17.4 \mathrm{q}$ \\
\hline 27 & $26.0 \mathrm{q}$ & $26.1 \mathrm{q}$ & $26.1 \mathrm{q}$ & $26.2 \mathrm{q}$ & $26.1 \mathrm{q}$ & $26.1 \mathrm{q}$ & $26.0 \mathrm{q}$ & $26.2 \mathrm{q}$ & $26.1 \mathrm{q}$ & $26.2 \mathrm{q}$ & $26.0 \mathrm{q}$ & $26.1 \mathrm{q}$ \\
\hline 28 & $176.4 \mathrm{~s}$ & $180.6 \mathrm{~s}$ & $176.6 \mathrm{~s}$ & $180.3 \mathrm{~s}$ & $176.6 \mathrm{~s}$ & $180.2 \mathrm{~s}$ & $176.5 \mathrm{~s}$ & $178.9 \mathrm{~s}$ & $176.6 \mathrm{~s}$ & $180.3 \mathrm{~s}$ & $176.3 \mathrm{~s}$ & $179.9 \mathrm{~s}$ \\
\hline 29 & $181.1 \mathrm{~s}$ & $181.0 \mathrm{~s}$ & $28.3 \mathrm{q}$ & $28.4 \mathrm{q}$ & $73.7 \mathrm{t}$ & $73.9 \mathrm{t}$ & $33.0 \mathrm{q}$ & $33.2 \mathrm{q}$ & $73.6 \mathrm{t}$ & $73.9 \mathrm{t}$ & $181.1 \mathrm{~s}$ & $181.1 \mathrm{~s}$ \\
\hline 30 & $\begin{array}{c}19.9 \mathrm{q} \\
28-O-\mathrm{glc}\end{array}$ & $20.0 \mathrm{q}$ & $65.4 \mathrm{t}$ & $65.6 \mathrm{t}$ & $19.7 \mathrm{q}$ & $19.7 \mathrm{q}$ & $23.6 \mathrm{q}$ & $23.8 \mathrm{q}$ & $19.7 \mathrm{q}$ & $19.8 \mathrm{q}$ & $19.9 \mathrm{q}$ & $20.0 \mathrm{q}$ \\
\hline $1^{\prime}$ & $95.7 \mathrm{~d}$ & & $95.7 \mathrm{~d}$ & & $95.7 \mathrm{~d}$ & & $95.6 \mathrm{~d}$ & & $95.6 \mathrm{~d}$ & & $95.7 \mathrm{~d}$ & \\
\hline $2^{\prime}$ & $73.8 \mathrm{~d}$ & & $73.8 \mathrm{~d}$ & & $73.9 \mathrm{~d}$ & & $73.8 \mathrm{~d}$ & & $73.9 \mathrm{~d}$ & & $73.8 \mathrm{~d}$ & \\
\hline $3^{\prime}$ & $78.6 \mathrm{~d}$ & & $78.7 \mathrm{~d}$ & & $78.7 \mathrm{~d}$ & & $78.6 \mathrm{~d}$ & & $78.7 \mathrm{~d}$ & & $78.7 \mathrm{~d}$ & \\
\hline $4^{\prime}$ & $70.8 \mathrm{~d}$ & & $70.9 \mathrm{~d}$ & & $70.8 \mathrm{~d}$ & & $70.8 \mathrm{~d}$ & & $70.8 \mathrm{~d}$ & & $70.7 \mathrm{~d}$ & \\
\hline $5^{\prime}$ & $77.9 \mathrm{~d}$ & & $77.9 \mathrm{~d}$ & & $78.0 \mathrm{~d}$ & & $78.0 \mathrm{~d}$ & & $78.0 \mathrm{~d}$ & & $78.0 \mathrm{~d}$ & \\
\hline $6^{\prime}$ & $\begin{array}{c}69.2 \mathrm{t} \\
6^{\prime}-O-\mathrm{glc}\end{array}$ & & $69.4 \mathrm{t}$ & & $69.2 \mathrm{t}$ & & $69.1 \mathrm{t}$ & & $69.2 \mathrm{t}$ & & $69.2 \mathrm{t}$ & \\
\hline $1^{\prime \prime}$ & $104.8 \mathrm{~d}$ & & $105.0 \mathrm{~d}$ & & $104.9 \mathrm{~d}$ & & $104.8 \mathrm{~d}$ & & $104.9 \mathrm{~d}$ & & $104.9 \mathrm{~d}$ & \\
\hline $2^{\prime \prime}$ & $75.2 \mathrm{~d}$ & & $75.3 \mathrm{~d}$ & & $75.3 \mathrm{~d}$ & & $75.3 \mathrm{~d}$ & & $75.3 \mathrm{~d}$ & & $75.3 \mathrm{~d}$ & \\
\hline $3^{\prime \prime}$ & $76.5 \mathrm{~d}$ & & $76.5 \mathrm{~d}$ & & $76.5 \mathrm{~d}$ & & $76.5 \mathrm{~d}$ & & $76.5 \mathrm{~d}$ & & $76.5 \mathrm{~d}$ & \\
\hline $4^{\prime \prime}$ & $78.3 \mathrm{~d}$ & & $78.4 \mathrm{~d}$ & & $78.3 \mathrm{~d}$ & & $78.3 \mathrm{~d}$ & & $78.3 \mathrm{~d}$ & & $78.2 \mathrm{~d}$ & \\
\hline $5^{\prime \prime}$ & $77.1 \mathrm{~d}$ & & $77.2 \mathrm{~d}$ & & $77.2 \mathrm{~d}$ & & $77.1 \mathrm{~d}$ & & $77.2 \mathrm{~d}$ & & $77.1 \mathrm{~d}$ & \\
\hline $6^{\prime \prime}$ & $\begin{array}{c}61.2 \mathrm{t} \\
4^{\prime \prime}-O \text {-rha }\end{array}$ & & $61.4 \mathrm{t}$ & & $61.3 \mathrm{t}$ & & $61.5 \mathrm{t}$ & & $61.3 \mathrm{t}$ & & $61.3 \mathrm{t}$ & \\
\hline 1 & $102.7 \mathrm{~d}$ & & $102.8 \mathrm{~d}$ & & $102.7 \mathrm{~d}$ & & $102.7 \mathrm{~d}$ & & $102.7 \mathrm{~d}$ & & $102.7 \mathrm{~d}$ & \\
\hline 2 & $72.5 \mathrm{~d}$ & & $72.6 \mathrm{~d}$ & & $72.6 \mathrm{~d}$ & & $72.5 \mathrm{~d}$ & & $72.6 \mathrm{~d}$ & & $72.5 \mathrm{~d}$ & \\
\hline 3 & $72.7 \mathrm{~d}$ & & $72.8 \mathrm{~d}$ & & $72.8 \mathrm{~d}$ & & $72.7 \mathrm{~d}$ & & $72.8 \mathrm{~d}$ & & $72.7 \mathrm{~d}$ & \\
\hline 4 & $73.9 \mathrm{~d}$ & & $74.0 \mathrm{~d}$ & & $74.0 \mathrm{~d}$ & & $73.9 \mathrm{~d}$ & & $74.0 \mathrm{~d}$ & & $73.9 \mathrm{~d}$ & \\
\hline 5 & $70.3 \mathrm{~d}$ & & $70.4 \mathrm{~d}$ & & $70.3 \mathrm{~d}$ & & $70.3 \mathrm{~d}$ & & $70.3 \mathrm{~d}$ & & $70.3 \mathrm{~d}$ & \\
\hline 6 & $18.5 \mathrm{q}$ & & $18.5 \mathrm{q}$ & & $18.5 \mathrm{q}$ & & $18.6 \mathrm{q}$ & & $18.5 \mathrm{q}$ & & $18.5 \mathrm{q}$ & \\
\hline
\end{tabular}

Multiplicities were obtained from DEPT experiments.

thine proton $[\delta 3.42(1 \mathrm{H}, \mathrm{dd}, J=3.4,13.9 \mathrm{~Hz})]$. The EI-MS spectrum of 5a showed a molecular ion at $\mathrm{m} / \mathrm{z} 486$ and fragments at $m / z 264$ and 219 . On comparison of the ${ }^{13} \mathrm{C}$-NMR spectra of $\mathbf{5}$ with acanjaposide B ( $3 \beta$-hydroxyl-23-oxo-olean12, 20(29)-dien-28-oic acid glycoside) $)^{1)}$ and acanjaposide $\mathrm{F}(3)$, the signals due to $\mathrm{A} / \mathrm{B} / \mathrm{C} / \mathrm{D}$ ring carbons were found the same as those of acanjaposide $\mathrm{B}$, and the signals due to $\mathrm{E}$ ring carbons were the same as those of $\mathbf{3}$. Furthermore, the correlation cross peaks from $\mathrm{H}-30[\delta 1.09(3 \mathrm{H}, \mathrm{s})]$ to $\mathrm{H}-18$ $[\delta 3.31(1 \mathrm{H}, \mathrm{dd}, J=3.2,12.0 \mathrm{~Hz})$, and from $\mathrm{H}-24[\delta 1.36$ $(3 \mathrm{H}, \mathrm{s})]$ to $\mathrm{H}-25[\delta 0.94(3 \mathrm{H}, \mathrm{s})]$ were observed in the NOESY spectrum of $\mathbf{5}$. Therefore, acanjaposide H (5) was determined to be $3 \beta$-hydroxyl-23-oxo-olean-12-en-28-oic acid $28-O$ - $\alpha$-L-rhamnopyranosyl-( $1 \rightarrow 4)-\beta$-D-glucopyranosyl$(1 \rightarrow 6)]-\beta$-D-glucopyranoside.

Compound 6 (acanjaposide I) exhibited a quasimolecular ion at $m / z 993.4661$ due to $[\mathrm{M}+\mathrm{Na}]^{+}$corresponding to the molecular formula $\mathrm{C}_{48} \mathrm{H}_{74} \mathrm{O}_{20}$ in the HR-FAB-MS. The ${ }^{13} \mathrm{C}$ NMR spectrum of $\mathbf{6}$ showed 48 signals, which were similar to those of $\mathbf{5}$ except for an aglycone E-ring moiety assignable to C-17, C-20, and C-29 (Table 1). The carbon signals of the E-ring moiety, including the signal at $\delta 181.0$ ascribable to carboxylic acid were similar to those of acanjaposide D (1). Its aglycone 6a, obtained by alkali hydrolysis, showed a qua- 


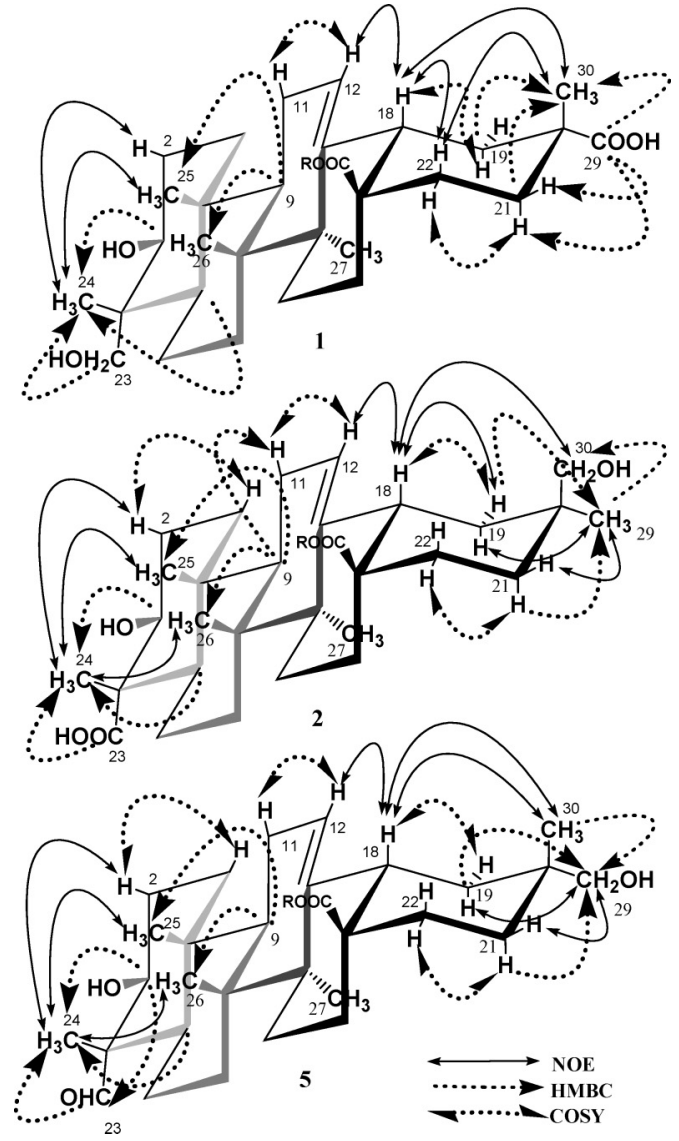

Fig. 1. Selected COSY, HMBC and NOE Data

simolecular ion at $\mathrm{m} / \mathrm{z} 523$ due to $[\mathrm{M}+\mathrm{Na}]^{+}$in the positive FAB-MS, and a molecular ion peak at $\mathrm{m} / \mathrm{z} 502$ along with characteristic fragment peaks at $\mathrm{m} / z 264$ and 233 in EI-MS corresponding to the molecular formula $\mathrm{C}_{30} \mathrm{H}_{44} \mathrm{O}_{6}$. Thus, $6 \mathbf{a}$ was putatively identified as a $3 \beta$-hydroxyl-23-oxo-olean-12en-28,29-dioic acid by NMR. Its sugar moiety was the same as that of compounds $\mathbf{1}-\mathbf{5}$ based on sugar analysis and NMR data. Therefore, acanjaposide I (6) was established as $3 \beta$-hydroxyl-olean-12-en-28,29-dioic acid 28- $O$ - $\alpha$-L-rhamnopyranosyl-( $1 \rightarrow 4)$ - $\beta$-D-glucopyranosyl-( $1 \rightarrow 6)]-\beta$-D-glucopyranoside.

From a chemotaxonomic point of view, it was of interest to note that the various oleanene analogues possessing a dicarboxylic acid $(1,2,4,5,6)$, or an aldehyde $(3,4)$ of $\beta$-amyrin skeleton, have been found only in the Acanthopanax species. $^{6-12)}$

\section{Experimental}

General Experimental Procedures The equipment used, the plant material, the extraction and isolation of the saponins, and methods used for alkaline and acidic hydrolysis, sapogenin mixture chromatography, and sugar characterizations were as described previously. ${ }^{1)}$ Compounds $\mathbf{1}-\mathbf{6}$ were obtaned from the $80 \% \mathrm{MeOH}$ fraction of Diaion-HP 20 described previously, ${ }^{1)}$ followed by silica gel column chromatography using $\mathrm{CHCl}_{3}-\mathrm{MeOH}-\mathrm{H}_{2} \mathrm{O}$ $(8: 2: 0.2 \rightarrow 7: 3: 0.2)$ as eluent and reverse-phase column chromatography using a $50 \%$ to $100 \% \mathrm{MeOH}$ gradient. In addition to acanjaposide $\mathrm{A}, \mathrm{B}$, and C previously reported, acanjaposide D (1, 53.5 mg), E (2, $19.5 \mathrm{mg}), \mathrm{F}(\mathbf{3}$, $467.4 \mathrm{mg}), \mathrm{G}(\mathbf{4}, 250.9 \mathrm{mg}), \mathrm{H}(\mathbf{5}, 425.3 \mathrm{mg})$, and I $(\mathbf{6}, 110.1 \mathrm{mg})$ were obtained during the present study.

Acanjaposide D (1): A white powder, $[\alpha]_{\mathrm{D}}^{25}-12.0^{\circ}(c=0.61, \mathrm{MeOH})$ Positive HR-FAB-MS $m / z$ : $995.4826\left(\mathrm{M}+\mathrm{Na}^{+}\right.$, Calcd for $\mathrm{C}_{48} \mathrm{H}_{76} \mathrm{O}_{20} \cdot \mathrm{Na}$ :
995.4828). ${ }^{1} \mathrm{H}-\mathrm{NMR}\left(500 \mathrm{MHz}\right.$, pyridine- $\left.d_{5}\right) \delta: 1.00(3 \mathrm{H}, \mathrm{s}, \mathrm{H}-25), 1.05$ (3H, s, H-24), 1.13 (3H, s, H-26), 1.18 (3H, s, H-27), 1.31 (1H, m, H-7b), $1.43(3 \mathrm{H}, \mathrm{s}, \mathrm{H}-30), 1.68(3 \mathrm{H}, \mathrm{d}, J=6.1 \mathrm{~Hz}, \mathrm{Rha} \mathrm{H}-6), 2.23(1 \mathrm{H}, \mathrm{m}, \mathrm{H}-15 \mathrm{a})$, $2.53(1 \mathrm{H}, \mathrm{dd}, J=14.0,14.0 \mathrm{~Hz}, \mathrm{H}-19 \mathrm{a}), 3.30(1 \mathrm{H}, \mathrm{dd}, J=3.2,13.7 \mathrm{~Hz}, \mathrm{H}-18)$, $3.68(1 \mathrm{H}, \mathrm{d}, J=10.3 \mathrm{~Hz}, \mathrm{H}-23 \mathrm{~b}), 3.62(1 \mathrm{H}, \mathrm{d}, J=10.7 \mathrm{~Hz}, \mathrm{Glc} \mathrm{H}-5), 3.68$ $(1 \mathrm{H}, \mathrm{d}, J=10.3 \mathrm{~Hz}, \mathrm{H}-23 \mathrm{~b}), 4.98\left(1 \mathrm{H}, \mathrm{d}, J=7.9 \mathrm{~Hz}, \mathrm{Glc}^{\prime} \mathrm{H}-1\right), 5.49(1 \mathrm{H}, \mathrm{br} \mathrm{s}$, $\mathrm{H}-12), 5.82(1 \mathrm{H}, \mathrm{s}$, Rha $\mathrm{H}-1), 6.27(1 \mathrm{H}, J=8.6 \mathrm{~Hz}$, Glc $\mathrm{H}-1) .{ }^{13} \mathrm{C}-\mathrm{NMR}$ : Table 1.

Acanjaposide E (2): A white powder; $[\alpha]_{\mathrm{D}}^{25}-3.6^{\circ}(c=1.08, \mathrm{MeOH})$. Positive FAB-MS $m / z$ : $996\left(\mathrm{M}+\mathrm{Na}^{+}\right)$. Positive HR-FAB-MS $\mathrm{m} / \mathrm{z} 995.4821$ (Calcd for $\mathrm{C}_{48} \mathrm{H}_{76} \mathrm{O}_{20} \cdot \mathrm{Na}$ : 995.4828). ${ }^{1} \mathrm{H}-\mathrm{NMR}\left(500 \mathrm{MHz}\right.$, pyridine- $\left.d_{5}\right) \delta$ : $1.00(3 \mathrm{H}, \mathrm{s}, \mathrm{H}-25), 1.11$ (3H, s, H-26), $1.14(3 \mathrm{H}, \mathrm{s}, \mathrm{H}-29), 1.25(3 \mathrm{H}, \mathrm{s}, \mathrm{H}-$ 27), $1.65(3 \mathrm{H}, \mathrm{s}, \mathrm{H}-24), 1.69(3 \mathrm{H}, \mathrm{d}, J=6.1 \mathrm{~Hz}$, Rha H-6), $2.29(1 \mathrm{H}$, ddd, $J=3.1,13.4,13.4 \mathrm{~Hz}, \mathrm{H}-15 \mathrm{a}), 3.34(1 \mathrm{H}, \mathrm{dd}, J=6.7,11.6 \mathrm{~Hz}, \mathrm{H}-18), 3.63$ $(1 \mathrm{H}, \mathrm{d}, J=10.7 \mathrm{~Hz}$, Glc H-5), $3.80(1 \mathrm{H}, \mathrm{d}, J=10.4 \mathrm{~Hz}, \mathrm{H}-23 \mathrm{~b}), 3.84(1 \mathrm{H}, \mathrm{d}$, $J=10.4 \mathrm{~Hz}, \mathrm{H}-23 \mathrm{a}), 4.98\left(1 \mathrm{H}, \mathrm{d}, J=7.9 \mathrm{~Hz}, \mathrm{Glc}{ }^{\prime} \mathrm{H}-1\right), 5.44(1 \mathrm{H}, \mathrm{br} \mathrm{s}, \mathrm{H}-12)$, $5.84\left(1 \mathrm{H}, \mathrm{s}\right.$, Rha H-1), $6.22(1 \mathrm{H}, J=8.5 \mathrm{~Hz}, \mathrm{Glc} \mathrm{H}-1) .{ }^{13} \mathrm{C}-\mathrm{NMR}$ : Table 1.

Acanjaposide $\mathrm{F}(3)$ : A white powder, $[\alpha]_{\mathrm{D}}^{25}+1.9^{\circ}(c=0.60, \mathrm{MeOH})$. Positive HR-FAB-MS m/z: $995.4835\left(\mathrm{M}+\mathrm{Na}^{+}\right.$, Calcd for $\mathrm{C}_{48} \mathrm{H}_{76} \mathrm{O}_{20} \cdot \mathrm{Na}$ : 995.4828). ${ }^{1} \mathrm{H}-\mathrm{NMR}\left(500 \mathrm{MHz}\right.$, pyridine- $\left.d_{5}\right) \delta: 1.02(3 \mathrm{H}, \mathrm{s}, \mathrm{H}-25), 1.09$ $(3 \mathrm{H}, \mathrm{s}, \mathrm{H}-30), 1.14(3 \mathrm{H}, \mathrm{s}, \mathrm{H}-26), 1.22(3 \mathrm{H}, \mathrm{s}, \mathrm{H}-27), 1.65(3 \mathrm{H}, \mathrm{s}, \mathrm{H}-24)$, $1.71(3 \mathrm{H}, \mathrm{d}, J=6.1 \mathrm{~Hz}$, Rha H-6), 2.09 (1H, dd, $J=13.7,13.9 \mathrm{~Hz}, \mathrm{H}-19 \mathrm{a})$, $2.30(1 \mathrm{H}$, ddd $J=2.4,12.8,13.4 \mathrm{~Hz} \mathrm{H}-15 \mathrm{a}), 3.30(1 \mathrm{H}, \mathrm{dd}, J=3.2,13.7 \mathrm{~Hz}$, H-18), $3.52(2 \mathrm{H}, \mathrm{s}, \mathrm{H}-29), 3.63(1 \mathrm{H}, \mathrm{d}, J=10.7 \mathrm{~Hz}$, Glc H-5), $4.99(1 \mathrm{H}, \mathrm{d}$, $\left.J=7.9 \mathrm{~Hz}, \mathrm{Glc}{ }^{\prime} \mathrm{H}-1\right), 5.47(1 \mathrm{H}$, br s, H-12), $5.85(1 \mathrm{H}, \mathrm{s}, \mathrm{Rha} \mathrm{H}-1), 6.25(1 \mathrm{H}$, $J=7.9 \mathrm{~Hz}$, Glc H-1). ${ }^{13} \mathrm{C}-\mathrm{NMR}$ : Table 1 .

Acanjaposide $\mathrm{G}(4)$ : A white powder; $[\alpha]_{\mathrm{D}}^{25}+1.5^{\circ}(c=1.15$, MeOH). Positive HR-FAB-MS $m / z$ : $979.4905\left(\mathrm{M}+\mathrm{Na}^{+}\right.$, Calcd for $\mathrm{C}_{48} \mathrm{H}_{76} \mathrm{O}_{19} \cdot \mathrm{Na}$ : 979.4879). ${ }^{1} \mathrm{H}-\mathrm{NMR}\left(500 \mathrm{MHz}\right.$, pyridine- $\left.d_{5}\right) \delta: 0.87(3 \mathrm{H}, \mathrm{s}, \mathrm{H}-29), 0.89$ $(3 \mathrm{H}, \mathrm{s}, \mathrm{H}-30), 1.02(3 \mathrm{H}, \mathrm{s}, \mathrm{H}-25), 1.12(3 \mathrm{H}, \mathrm{s}, \mathrm{H}-26), 1.20$ (3H, s, H-27), $1.56(1 \mathrm{H}$, br d, $J=13.5 \mathrm{~Hz}, \mathrm{H}-6), 1.66(3 \mathrm{H}, \mathrm{s}, \mathrm{H}-24), 1.70(3 \mathrm{H}, \mathrm{d}, J=6.1 \mathrm{~Hz}$, Rha H-6), 2.28 (1H, ddd, $J=3.4,13.8,13.8 \mathrm{~Hz}, \mathrm{H}-15 \mathrm{a}), 3.18$ ( $1 \mathrm{H}, \mathrm{dd}, J=3.2$, $12.9 \mathrm{~Hz}, \mathrm{H}-18), 3.62(1 \mathrm{H}, \mathrm{d}, J=10.7 \mathrm{~Hz}, \mathrm{Glc} \mathrm{H}-5), 4.99(1 \mathrm{H}, \mathrm{d}, J=7.9 \mathrm{~Hz}$, Glc' H-1), $5.44(1 \mathrm{H}$, br s, H-12), $5.85(1 \mathrm{H}, \mathrm{s}, \mathrm{Rha} \mathrm{H}-1), 6.23(1 \mathrm{H}, J=8.6 \mathrm{~Hz}$, Glc H-1). ${ }^{13} \mathrm{C}-\mathrm{NMR}$ : Table 1.

Acanjaposide $\mathrm{H}(5)$ : A white powder; $[\alpha]_{\mathrm{D}}^{25}+3.4^{\circ}(c=0.54, \mathrm{MeOH})$. Positive HR-FAB-MS m/z: $979.4884\left(\mathrm{M}+\mathrm{Na}^{+}\right.$, Calcd for $\mathrm{C}_{48} \mathrm{H}_{76} \mathrm{O}_{19} \cdot \mathrm{Na}$ : 979.4878). ${ }^{1} \mathrm{H}-\mathrm{NMR}\left(500 \mathrm{MHz}\right.$, pyridine- $\left.d_{5}\right) \delta: 0.94(3 \mathrm{H}, \mathrm{s}, \mathrm{H}-25), 1.09$ $(3 \mathrm{H}, \mathrm{s}, \mathrm{H}-30), 1.10(3 \mathrm{H}, \mathrm{s}, \mathrm{H}-26), 1.25$ (3H, s, H-27), 1.36 (3H, s, H-24), $1.57(1 \mathrm{H}$, brd, $J=13.4 \mathrm{~Hz} \mathrm{H}-1 \mathrm{~b}), 1.70(3 \mathrm{H}, \mathrm{d}, J=6.1 \mathrm{~Hz}$, Rha H-6), 2.14 $(1 \mathrm{H}, \mathrm{dd}, J=14.7,14.7 \mathrm{~Hz}, \mathrm{H}-19 \mathrm{a}), 2.30(1 \mathrm{H}, \mathrm{ddd}, J=3.1,13.4,13.4 \mathrm{~Hz} \mathrm{H}-$ $15 \mathrm{a}), 3.31(1 \mathrm{H}, \mathrm{dd}, J=3.2,12.0 \mathrm{~Hz}, \mathrm{H}-18), 3.55(2 \mathrm{H}, \mathrm{s}, \mathrm{H}-29), 3.63(1 \mathrm{H}, \mathrm{d}$, $J=10.7 \mathrm{~Hz}$, Glc H-5), $4.99(1 \mathrm{H}, \mathrm{d}, J=7.8 \mathrm{~Hz}$, Glc' $\mathrm{H}-1), 5.47(1 \mathrm{H}$, br s, H12), $5.85\left(1 \mathrm{H}, \mathrm{s}\right.$, Rha H-1), $6.26\left(1 \mathrm{H}, J=7.9 \mathrm{~Hz}\right.$, Glc H-1). ${ }^{13} \mathrm{C}$-NMR: Table 1.

Acanjaposide I (6): A white powder; $[\alpha]_{\mathrm{D}}^{25}+3.6^{\circ}(c=0.56, \mathrm{MeOH})$. Positive HR-FAB-MS $m / z$ : $993.4661\left(\mathrm{M}+\mathrm{Na}^{+}\right.$, Calcd for $\mathrm{C}_{48} \mathrm{H}_{74} \mathrm{O}_{20} \cdot \mathrm{Na}$ : 993.4671). ${ }^{1} \mathrm{H}-\mathrm{NMR}\left(500 \mathrm{MHz}\right.$, pyridine- $\left.d_{5}\right) \delta: 0.94(3 \mathrm{H}, \mathrm{s}, \mathrm{H}-25), 1.09$ $(3 \mathrm{H}, \mathrm{s}, \mathrm{H}-26), 1.22(3 \mathrm{H}, \mathrm{s}, \mathrm{H}-27), 1.36$ (3H, s, H-24), $1.46(3 \mathrm{H}, \mathrm{s}, \mathrm{H}-30)$, $1.58(1 \mathrm{H}$, br d, $J=12.8 \mathrm{~Hz} \mathrm{H}-1 \mathrm{~b}), 1.71(3 \mathrm{H}, \mathrm{d}, J=6.1 \mathrm{~Hz}$, Rha H-6), 2.28 $(1 \mathrm{H}, \mathrm{ddd}, J=2.4,12.2,13.5 \mathrm{~Hz} \mathrm{H}-15 \mathrm{a}), 2.54(1 \mathrm{H}, \mathrm{dd}, J=13.7,14.0 \mathrm{~Hz}, \mathrm{H}-$ 19a), $3.32(1 \mathrm{H}, \mathrm{dd}, J=4.3,14.0 \mathrm{~Hz}, \mathrm{H}-18), 3.62(1 \mathrm{H}, \mathrm{d}, J=10.7 \mathrm{~Hz}$, Glc H5), $4.99\left(1 \mathrm{H}, \mathrm{d}, J=7.9 \mathrm{~Hz}, \mathrm{Glc}^{\prime} \mathrm{H}-1\right), 5.49(1 \mathrm{H}, \mathrm{br} \mathrm{s}, \mathrm{H}-12), 5.85(1 \mathrm{H}, \mathrm{s}$, Rha $\mathrm{H}-1), 6.26\left(1 \mathrm{H}, J=7.9 \mathrm{~Hz}\right.$, Glc H-1). ${ }^{13} \mathrm{C}-\mathrm{NMR}$ : Table 1 .

Alkali Hydrolysis of Compounds $\mathbf{1}-\mathbf{6}$ These compounds $(34.4 \mathrm{mg}$, $15.0 \mathrm{mg}, 48.0 \mathrm{mg}, 64.8 \mathrm{mg}, 52.3 \mathrm{mg}$, and $66.1 \mathrm{mg}$, respectively) were hydrolyzed with $2 \mathrm{ml}$ of $3 \% \mathrm{KOH}$, and then neutralized and purified by column chromatography $^{1)}$ to give compounds 1a $(17.3 \mathrm{mg}), \mathbf{2 a}(8.0 \mathrm{mg}), 3 \mathbf{3}$ $(20.1 \mathrm{mg})$, and $\mathbf{4 a}(31.4 \mathrm{mg}), \mathbf{5 a}(16.0 \mathrm{mg})$, and $\mathbf{6 a}(31.1 \mathrm{mg})$, as their respective aglycone.

Sugar Analysis Each compound (about $3 \mathrm{mg}$ ) was hydrolyzed with $2 \mathrm{ml}$ of $2 \mathrm{~N} \mathrm{HCl}$ in $\mathrm{H}_{2} \mathrm{O}$ for $4 \mathrm{~h}$ at $80^{\circ} \mathrm{C}$, which was followed by neutralization with $2 \mathrm{~N} \mathrm{NaOH}$ in $\mathrm{H}_{2} \mathrm{O}$ and extraction with $\mathrm{CHCl}_{3}$. The aqueous layer was evaporated to dryness under vacuum. The residue was treated with the silylation reagent (Sylon HTP kit, Supelco) for $20 \mathrm{~min}$ at room temperature. After removing the excess reagent and organic solvent by condensation, the persilylated products were dissolved in hexane, and this solution was subjected to the GC analysis: column, fused silica capillary column (OV-17, $0.32 \times 30 \mathrm{~cm}$ ); detector, FID; column temperature, gradient of $100^{\circ} \mathrm{C}$ to $250^{\circ} \mathrm{C}$ at $15^{\circ} \mathrm{C} / \mathrm{min}$. The retention times of persilylated glucose and rhamnose were found to be 10.8 and $7.01 \mathrm{~min}$, respectively, when compared with the standard solutions prepared by the same reaction from the standard monosaccharides.

Acanjapogenin D (1a): White powder; $[\alpha]_{\mathrm{D}}^{25}+56.2^{\circ}(c=0.55, \mathrm{MeOH})$. 
EI-MS $m / z(\%): 502\left(\mathrm{M}^{+}, 2\right), 488$ (4), 278 (27), 264 (74), 233 (100), 206 (24), 201 (50), 187 (34), 175 (26), 159 (11), 119 (12), 105 (12). ${ }^{1} \mathrm{H}-\mathrm{NMR}$ $\left(500 \mathrm{MHz}\right.$, pyridine- $\left.d_{5}\right) \delta: 0.97(3 \mathrm{H}, \mathrm{s}, \mathrm{H}-25), 1.05(3 \mathrm{H}, \mathrm{s}, \mathrm{H}-24), 1.05(3 \mathrm{H}$, s, H-26), $1.24(3 \mathrm{H}, \mathrm{s}, \mathrm{H}-27), 1.57$ (3H, s, H-30), $1.76(1 \mathrm{H}, \mathrm{m}, \mathrm{H}-9), 2.31$ $(1 \mathrm{H}, \mathrm{ddd}, J=3.9,13.4,13.4 \mathrm{~Hz}, \mathrm{H}-21 \mathrm{a}), 2.58(1 \mathrm{H}, \mathrm{dd}, J=13.7,14.0 \mathrm{~Hz}, \mathrm{H}-$ $19 \mathrm{a}), 3.44(1 \mathrm{H}, \mathrm{dd}, J=3.7,13.7 \mathrm{~Hz}, \mathrm{H}-18), 3.71(1 \mathrm{H}, \mathrm{d}, J=11.0 \mathrm{~Hz}, \mathrm{H}-23 \mathrm{~b})$, $4.16(1 \mathrm{H}, \mathrm{d}, J=11.0 \mathrm{~Hz}, \mathrm{H}-23 \mathrm{a}), 4.18(1 \mathrm{H}, \mathrm{dd}, J=5.2,11.0 \mathrm{~Hz}, \mathrm{H}-3), 5.58$ (1H, br s, H-12). ${ }^{13} \mathrm{C}-\mathrm{NMR}$ : Table 1.

Acanjapogenin E (2a): White powder; $[\alpha]_{\mathrm{D}}^{25}+55.1^{\circ}(c=0.50, \mathrm{MeOH})$. Positive HR-FAB-MS m/z: $525.3192\left(\mathrm{M}+\mathrm{Na}^{+}\right.$, Calcd for $\mathrm{C}_{30} \mathrm{H}_{46} \mathrm{O}_{6} \cdot \mathrm{Na}$ : 525.3192). ${ }^{1} \mathrm{H}-\mathrm{NMR}\left(500 \mathrm{MHz}\right.$, pyridine- $\left.d_{5}\right) \delta: 0.96(3 \mathrm{H}, \mathrm{s}, \mathrm{H}-25), 1.02$ $(3 \mathrm{H}, \mathrm{s}, \mathrm{H}-26), 1.20(3 \mathrm{H}, \mathrm{s}, \mathrm{H}-29), 1.30(3 \mathrm{H}, \mathrm{s}, \mathrm{H}-27), 1.64(3 \mathrm{H}, \mathrm{s}, \mathrm{H}-24)$, $2.04(1 \mathrm{H}$, ddd, $J=6.1,6.1,11.6 \mathrm{~Hz}, \mathrm{H}-5), 3.44(1 \mathrm{H}, \mathrm{dd}, J=6.4,11.9 \mathrm{~Hz}, \mathrm{H}-$ 18), 3.92 (1H, d, $J=10.4 \mathrm{~Hz}, \mathrm{H}-30 \mathrm{~b}), 3.99$ (1H, d, $J=10.4 \mathrm{~Hz}, \mathrm{H}-30 \mathrm{a}), 4.67$ $(1 \mathrm{H}, \mathrm{dd}, J=7.9,7.9 \mathrm{~Hz}, \mathrm{H}-3), 5.51(1 \mathrm{H}$, br s, $\mathrm{H}-12) .{ }^{13} \mathrm{C}-\mathrm{NMR}$ : Table 1.

Acanjapogenin F (3a): White powder; $[\alpha]_{\mathrm{D}}^{25}+73.5^{\circ}(c=0.68, \mathrm{MeOH})$, Positive FAB-MS $m / z$ : $525\left(\mathrm{M}+\mathrm{Na}^{+}, \mathrm{C}_{30} \mathrm{H}_{44} \mathrm{O}_{6} \cdot \mathrm{Na}\right)$. EI-MS $m / z(\%): 502$ $\left(\mathrm{M}^{+}, 1\right), 264$ (36), 233 (100), 219 (21), 201 (78), 187 (42), 173 (24), 159 (31), 131 (29), 119 (27), $105(42) .{ }^{1} \mathrm{H}-\mathrm{NMR}\left(500 \mathrm{MHz}\right.$, pyridine- $\left.d_{5}\right) \delta: 0.98$ (3H, s, H-25), $1.04(3 \mathrm{H}, \mathrm{s}, \mathrm{H}-26), 1.22(3 \mathrm{H}, \mathrm{s}, \mathrm{H}-30), 1.27$ (3H, s, H-27), $1.40(1 \mathrm{H}, \mathrm{brd}, J=12.2 \mathrm{~Hz}, \mathrm{H}-21 \mathrm{~b}), 1.51(1 \mathrm{H}, \mathrm{dd}, J=3.4,13.2 \mathrm{~Hz}, \mathrm{H}-19 \mathrm{~b})$, $1.64(3 \mathrm{H}, \mathrm{s}, \mathrm{H}-24), 3.42(1 \mathrm{H}, \mathrm{dd}, J=3.4,13.6 \mathrm{~Hz}, \mathrm{H}-18), 3.59(2 \mathrm{H}, \mathrm{br} \mathrm{s}, \mathrm{H}-$ 29), $4.68(1 \mathrm{H}, \mathrm{dd}, J=7.9,7.9 \mathrm{~Hz}, \mathrm{H}-3), 5.54\left(1 \mathrm{H}\right.$, br s, H-12). ${ }^{13} \mathrm{C}-\mathrm{NMR}$ : Table 1.

Acanjapogenin G (4a): White powder; $[\alpha]_{\mathrm{D}}^{25}+52.6^{\circ}(c=0.46, \mathrm{MeOH})$. Positive FAB-MS $m / z: 509\left(\mathrm{M}+\mathrm{Na}^{+}, \mathrm{C}_{30} \mathrm{H}_{46} \mathrm{O}_{5} \cdot \mathrm{Na}\right)$. EI-MS $m / z(\%): 486$ $\left(\mathrm{M}^{+}, 1\right), 441$ (4), 248 (78), 203 (100), 189 (22), 175 (24), 161 (13), 147 (17), 133 (36), 119 (32), 105 (34). ${ }^{1} \mathrm{H}-\mathrm{NMR}\left(500 \mathrm{MHz}\right.$, pyridine- $\left.d_{5}\right) \delta: 0.94$ $(3 \mathrm{H}, \mathrm{s}, \mathrm{H}-29), 0.98$ (3H, s, H-25), $1.01(3 \mathrm{H}, \mathrm{s}, \mathrm{H}-30), 1.02(3 \mathrm{H}, \mathrm{s}, \mathrm{H}-26)$, $1.13(1 \mathrm{H}, \mathrm{brd}, J=12.2 \mathrm{~Hz}, \mathrm{H}-15 \mathrm{~b}), 1.25(3 \mathrm{H}, \mathrm{s}, \mathrm{H}-27), 1.63$ (3H, s, H-24), $3.29(1 \mathrm{H}, \mathrm{dd}, J=3.7,13.7 \mathrm{~Hz}, \mathrm{H}-18), 4.67(1 \mathrm{H}, \mathrm{dd}, J=6.9,8.6 \mathrm{~Hz}, \mathrm{H}-3), 5.50$ $(1 \mathrm{H}$, br s, $\mathrm{H}-12) .{ }^{13} \mathrm{C}-\mathrm{NMR}$ : Table 1 .

Acanjapogenin $\mathrm{H}(\mathbf{5 a})$ : White powder; $[\alpha]_{\mathrm{D}}^{25}+63.4^{\circ}(c=0.50, \mathrm{MeOH})$, Positive FAB-MS $m / z$ : $509\left(\mathrm{M}+\mathrm{Na}^{+}, \mathrm{C}_{30} \mathrm{H}_{46} \mathrm{O}_{5} \cdot \mathrm{Na}\right)$. EI-MS $m / z(\%): 486$ $\left(\mathrm{M}^{+}, 2\right), 456$ (5), 441 (2), 264 (51), 233 (100), 219 (15), 201 (67), 187 (32), 175 (19), 159 (22), 119 (29), 105 (33). ${ }^{1} \mathrm{H}-\mathrm{NMR}\left(500 \mathrm{MHz}\right.$, pyridine- $\left.d_{5}\right) \delta$ : $0.90(3 \mathrm{H}, \mathrm{s}, \mathrm{H}-25), 1.00(3 \mathrm{H}, \mathrm{s}, \mathrm{H}-26), 1.22(3 \mathrm{H}, \mathrm{s}, \mathrm{H}-30), 1.30(3 \mathrm{H}, \mathrm{s}, \mathrm{H}-$ 27), $1.35(3 \mathrm{H}, \mathrm{s}, \mathrm{H}-24), 1.57(1 \mathrm{H}, \mathrm{brd}, J=12.8 \mathrm{~Hz}, \mathrm{H}-1 \mathrm{~b}), 3.44(1 \mathrm{H}, \mathrm{dd}$, $J=3.4,13.9 \mathrm{~Hz}, \mathrm{H}-18), 3.60(2 \mathrm{H}, \mathrm{s}, \mathrm{H}-29), 4.06(1 \mathrm{H}, \mathrm{dd}, J=7.3,8.6 \mathrm{~Hz}, \mathrm{H}-$
3), 5.23 (1H, br s, H-12). ${ }^{13} \mathrm{C}-\mathrm{NMR}$ : Table 1.

Acanjapogenin I (6a): White powder; $[\alpha]_{\mathrm{D}}^{25}+49.2^{\circ}(c=0.48, \mathrm{MeOH})$, Positive FAB-MS $m / z$ : $523\left(\mathrm{M}+\mathrm{Na}^{+}, \mathrm{C}_{30} \mathrm{H}_{44} \mathrm{O}_{6} \cdot \mathrm{Na}\right) .{ }^{1} \mathrm{H}-\mathrm{NMR}(500 \mathrm{MHz}$, pyridine- $\left.d_{5}\right) \delta: 0.90(3 \mathrm{H}, \mathrm{s}, \mathrm{H}-25), 0.99(3 \mathrm{H}, \mathrm{s}, \mathrm{H}-26), 1.28(3 \mathrm{H}, \mathrm{s}, \mathrm{H}-27)$, $1.35(3 \mathrm{H}, \mathrm{s}, \mathrm{H}-24), 1.58(3 \mathrm{H}, \mathrm{s}, \mathrm{H}-30), 2.32(1 \mathrm{H}, \mathrm{ddd}, J=3.2,12.5,14.0 \mathrm{~Hz}$, $\mathrm{H}-21 \mathrm{a}), 2.59(1 \mathrm{H}, \mathrm{dd}, J=14.0,14.0 \mathrm{~Hz} \mathrm{H}-19 \mathrm{a}), 3.43(1 \mathrm{H}, \mathrm{dd}, J=3.7$, $14.0 \mathrm{~Hz}, \mathrm{H}-18), 4.07(1 \mathrm{H}, \mathrm{dd}, J=6.7,7.9 \mathrm{~Hz}, \mathrm{H}-3), 5.55(1 \mathrm{H}$, br s, H-12). ${ }^{13} \mathrm{C}-\mathrm{NMR}$ : Table 1.

\section{References}

1) Park S. Y., Chang S. Y., Oh O. J., Yook C. S., Nohara T., Phytochemistry, 59, 379-384 (2002).

2) Budzikiewicz H., Wilson J. M., Djerassi C., J. Am. Chem. Soc., 85, 3688-3699 (1963).

3) Shojima K., Arai Y., Masuda K., Takase Y., Ageta T., Ageta H., Chem. Pharm. Bull., 40, 1683-1690 (1992).

4) Mahato S., Kunda A., Phytochemistry, 37, 1517-1575 (1995).

5) Kasei R., Okihara M., Asakawa J., Mizukani K., Tanaka O., Tetrahedron, 35, 1427-1432 (1979).

6) Shao C.-J., Kasei R., Xu J.-D., Tanaka O., Chem. Pharm. Bull., 37, $42-45$ (1989).

7) Kohda H., Tanaka S., Yamaoka Y., Chem. Pharm. Bull., 38, 33803383 (1990).

8) Miyakoshi M., Isoda S., Ida Y., Shoji J., Phytochemistry, 34, 15991602 (1993).

9) Miyakoshi M., Isoda S., Satoh H., Hirai Y., Shoji J., Ida Y., Phytochemistry, 46, 1255-1259 (1997).

10) Chang S. Y., Yook C. S., Nohara T., Chem. Pharm. Bull., 46, 163165 (1998).

11) Park S. Y., Chang S. Y., Yook C. S., Nohara T., J. Nat. Prod., 63, $1630-1633$ (2000).

12) Yook C. S., Liu X. Q., Chang S. Y., Park S. Y., Nohara T., Chem. Pharm. Bull., 50, 1383-1385 (2002).

13) Doddrell D. M., Khong P. W., Lewis K. G., Tetrahedron Lett., 15, 2371-2384 (1974).

14) Ikuda A., J. Nat. Prod., 52, 623-628 (1989).

15) Yu S. S., Yu D. Q., Liang X. T., Phytochemistry, 38, 695-698 (1995).

16) Oshima Y., Ohsawa T., Hikino H., Planta Medica, 50, 43-47 (1984). 